\title{
Development status and application of neuronavigation system
}

\author{
Qi Zheng ${ }^{1}$, Chaoyue Liu ${ }^{2}$, Qiuling Pan ${ }^{3}$, Jincai Chang ${ }^{4}$, Jianzhong Cui ${ }^{5}$ \\ ${ }_{1,2,3,4}$ College of Sciences, North China University of Science and Technology, Tangshan, 063210, China \\ ${ }^{4} 3 \mathrm{D}$ Modeling and Application Innovation Laboratory, North China University of Science and Technology, \\ Tangshan, 063210, China \\ ${ }^{5}$ Department of Neurosurgery, Tangshan Gongren Hospital, Tangshan, 063000, China \\ ${ }^{4,5}$ Corresponding author \\ E-mail: ${ }^{1}$ billy0516z@163.com, ${ }^{2}$ lcy970306@163.com, ${ }^{3}$ pql_ncst@163.com, ${ }^{4}$ jincai@ncst.edu.cn, \\ 5zhcui0001@163.com
}

Received 23 December 2019; received in revised form 30 January 2020; accepted 25 February 2020 DOI https://doi.org/10.21595/chs.2020.21260

Check for updates

Copyright (C) 2020 Qi Zheng, et al. This is an open access article distributed under the Creative Commons Attribution License, which permits unrestricted use, distribution, and reproduction in any medium, provided the original work is properly cited.

\begin{abstract}
The neuronavigation system is a combination of navigation technology and neurosurgery. It can be used to assist in neurosurgery through three-dimensional reconstruction of medical image data, extraction of lesions, optimal surgical path planning, tracking and positioning of surgical instruments, and real-time intraoperative display. Accurate and maximal treatment of lesions, while effectively avoiding secondary injuries to patients during surgery. Therefore, the development and application of neuronavigation systems are reviewed.
\end{abstract}

Keywords: neuronavigation, navigation technology, real-time display, clinical application.

\section{Introduction}

In 2019, approximately $321,670(53 \%)$ men and 285,210 (47 \%) women in the U.S. died from malignant tumors in the brain and other areas of the central nervous system. A total of $1,806,590$ new cancer cases and 606,520 deaths are expected in the US in 2020, which is about 4,950 new cases and more than 1,600 deaths each day. Among them, young people between the ages of 15 and 39 will have 89,500 new cancer cases and 9,270 cancer deaths. In the United States, the number of new deaths associated with brain tumors is close to 12,760 per year. The average survival time of patients with low-grade glioma is about 7.5 years, while the average survival time of most patients with malignant glioma is about 1 year [1]. At present, there is no accepted treatment for most brain tumors. Surgical resection to reduce the number of tumor cells in patients with low-grade gliomas can extend patient survival $[2,3]$. Malignant tumors can be relieved by surgical resection. Even after radiation therapy and chemotherapy $[4,5]$, the survival rate is still low. Most primary brain tumors grow by infiltration of healthy tissue. Therefore, after surgical resection, the tumor tissue can be visually observed to be residual but cannot be removed, which greatly affects the patient's prognosis. Therefore, a major challenge in neurosurgical oncology is to achieve maximum tumor resection while avoiding neurological deficits after surgery. Neurosurgeons face many problems during tumor resection, such as: (1) try to remove tumor tissue; (2) minimize the removal of healthy tissue; (3) avoid destroying important anatomical structures; (4) know when to stop the resection process. Therefore, the development of intraoperative imaging systems can help improve the process of intracranial neurosurgery [6]. With the advent of big data, the rapid development of artificial intelligence and human-computer interaction and other subjects, the neuronavigation system has become an indispensable technology in surgery, and it is becoming more and more important. The neuronavigation system can realize preoperative surgery. Planning, reconstructing medical images based on data, and performing a certain degree of virtual demonstration of pre-operative operations before surgery, making it more fully prepared and improving the success rate of the operation; secondly, the navigation system provides real-time display during the operation. It can help physicians find surgical targets more accurately and quickly and accurately. At the same time, doctors can adjust 
the surgical path to different degrees through the spatial position relationship between lesions and healthy brain parenchyma, which greatly solves the problem of neurosurgeons in traditional surgery. The problems encountered in this method have improved the accuracy and completion rate of the operation. Finally, the surgical navigation system also established a special database to store the preoperative, intraoperative and postoperative data of the patient, so as to better achieve patient care and good prognosis.

At present, the neuronavigation system has been perfected, and has achieved great results in clinical applications. Among them, it is mainly divided into two types of navigation system equipment, which are optical navigation equipment and electromagnetic navigation equipment. Its representative products are Stealth Station system of the American Medtronic company, the Stryke Navigation system of the German Stryker company, and the Stealth Station Axi-EM system of the Medtronic company [7-9]. In neurosurgery, due to the opening of the dura mater, resection of tumor cyst components, osmotic displacement, release of cerebrospinal fluid, etc. may cause the displacement of the lesion, which may occur within one hour after dura mater opening Displacement of anatomical displacements over a distance of $10 \mathrm{~mm}$, especially when the tumor is large, cystic, and deep or when the cerebrospinal fluid space with continuous, in this case, the accuracy of conventional neural navigation system will be seriously affected [10-15]. If the intraoperative lesions shift, it will affect the patient model that has been established before the operation. So, the migration of the lesions will bring certain challenges to the conventional neuronavigation system. This part of the problem needs further discussion. This part of the problem needs further discussion.

This article first introduces the development process of the neuronavigation system, and simulates the entire navigation system process through the combination of the NDI Passive Polaris system and the open source software 3D Slicer. The purpose is to measure and calculate the registration accuracy and application accuracy of the navigation system, and evaluate processing software and hardware for use in a clinical setting. At the same time, the applications of neural navigation systems in different fields are reviewed. Finally, the problems encountered in the experiment are summarized and solutions are given, as well as the future prospects of neural navigation systems.

\section{History of the development of neuronavigation systems}

The neuronavigation system is developed on the basis of traditional stereotactic technology and realizes frameless spatial positioning. It mainly combines computer medical imaging technology, artificial intelligence technology and micro-invasive surgery technology, which is a good solution to the preoperative planning and real-time display problems. It is mainly divided into robotic arm navigation system and digitizer system (infrared navigation, electromagnetic navigation and ultrasonic navigation), including several parts: model reconstruction, optimal path planning, registration, intraoperative navigation, etc. The navigation system dates back to 1881 . The brain measuring instrument invented by the Russian anatomist Zernov completed the first stereotactic surgery in human history. In 1908, brain stereotactic technology began to be studied, and the British Horsley and Clarke created the brain stereotactic technology and the first stereotactic frame are recognized as stereotactic pioneers. Although the traditional stereotactic technology can determine one or several spatial coordinates, the boundary of the lesion cannot be determined, and the accuracy is also poor. The rack is cumbersome and brings a lot of inconvenience to the operation. In 1947, the semi-bow head frame of Swedish scientist Leksell laid the foundation for stereotactic technology. In the same year, Spiegel et al. applied the stereotactic system to humans for the first time, and its appearance marked the rise of early surgical navigation systems [16]. With the development of imaging technology, after the 1970s, computerized tomography (CT) and magnetic resonance imaging technologies were successively introduced, accompanied by more intelligent stereotactic technology. In 1986, Dr. Roberts of the United States created the first. Surgical navigation system which was first applied to the clinic 
[17]. He combines computed tomography image data (CT) with ultrasound localization-guided surgical techniques for use under surgical microscopes, combining traditional stereotactic techniques with computer-aided (B-guided surgery) techniques, Although the system overcome the non-comprehensiveness of the traditional stereotactic techniques and abandoned the bulky stereo positioning frame, the accuracy needs to be improved and it is greatly affected by the environment. So that B-ultrasound guided surgery in the real-time display function is also missing [17]. Subsequently, in response to the above problems, Bernett and Reinhardt and others improved the ultrasonic technology and improved the accuracy to some extent. Since then, in the late 1980s, the robotic arm navigation system began to appear one after another. In 1987, Japan Watanabe invented the joint arm positioning system [18]. In 1988, the PUMA262-based stereotactic brain surgery robot system researched by Y. S. Kwoh of Canada has used in clinical operations. In 1996, S. A. Tebo of the United States studied the frameless stereotactic surgery system based on optical positioning. In 1997, The robotic surgery system based on brain surgery for parallel robot mechanism was studied by T. C. Lueth of Germany [19]. Since then, the neuronavigation system has developed rapidly, gradually realizing the function of intraoperative real-time navigation. It also has been applied to various neurosurgery including biopsy. The articulated arm can adapt in various environments but is not constrained by to the line of sight. So, it's not enough for surgery. In the early 1990 s, optical positioning systems and electromagnetic positioning systems began to develop. In 1992, Heilbrun et al. used ordinary and infrared cameras, which is based on the principle of trinocular and binocular machine vision for stereotactic orientation [20]. This method has high precision. It can track multiple targets and is small in size and easy to operate. But it is easily interfered by background light and radiation. In the same year, Kato used small magnetic field sensors which is based on the magnetic field modulation technology to test the 3D-position and azimuth of the probes, resin pointers or pipettes. He introduced $\mathrm{ct} / \mathrm{mri}$ stereotactic techniques into traditional open neurosurgery, where the magnetic field detector can be placed anywhere, but the downside is that the system is heavily influenced by metal [21]. After that, people have proposed improvements and innovations in the positioning system. In 2004, based on optical positioning technology, a two-dimensional positioning system which is capable of high-precision and real-time optical positioning for multiple light spots in space was developed. It has great significance for the development of optical two-dimensional measurement technology [22]. In 2016, Lin Qinyong proposed automatic real-time surgical registration technology in operation navigation based on binocular vision positioning technology and medical image processing and visualization technology, and precise positioning and puncture technology for liver cancer ablation. And it has been widely used in clinical practice [23]. In 2017, Xue Yuan's optimization-based 3D-DIC scattered speckle tracking method is more convenient to arrange than the spherical punctuation used in traditional infrared surgery and can meet the advantages of realtime tracking of small objects [24].

\section{The structure and operation principle of the neuronavigation system}

The neuronavigation system is mainly composed of surgical data acquisition, preoperative modeling and intraoperative navigation. Among them, the surgical data acquisition part mainly with scans for medical imaging equipment for patients (CT/CTA, MRI/MRA/DTI, DSA, PET). Coronal, sagittal, and axial navigation sequence images scanned, and the layer thickness requirement was within $3 \mathrm{~mm} .7$ to 10 Makers were attached to the patient's head before scanning, as landmarks for subsequent surgical path planning and registration of the navigation system. And then the doctor will measure and analyze the processed image to diagnose the condition.

\subsection{Preoperative modeling}

The preoperative modeling part is composed of high-precision computer. The main function is to enhance, segment and register the DICOM data scanned by patients (CT/CTA, 
MRI/MRA/DTI, DSA, PET). Using CTA image segmentation technology to build focus shape, blood vessel shape and skull model. Using MRI image segmentation method to reconstruct brain tissue, tumor, ventricular system, fiber bundle shape and important functional areas. And through the integration of the virtual three-dimensional model and the marker of the real entity, as well as the extraction of the long axis and the short axis of the focus, the optimal operation path will be finally planned.

\subsubsection{Import of data}

Clinical medicine includes many different types of medical images. At present, people use three-dimensional medical imaging technologies including computerized tomography (CT), CT angiography (CTA), and magnetic resonance imaging (MRI) to obtain pathological information of patients and anatomical information. These 3D data have good contrast, can describe the anatomical information of the lesion in detail, and therefore play a very important role in $3 \mathrm{D}$ reconstruction of the patient.

First, the patient's skull CT and DICOM data scanned by MRI workstations are imported into the open source software 3D Slicer. As shown in Fig. 1, which is a CT image of a hematoma in the brain, from the patient's coronary, sagittal, axial, and the 3D window, it can be clearly seen that the white area is the hematoma in the patient's brain. Fig. 2 shows the patient's MRI data. It can be clearly seen the patient's diseased area through the axial position, coronal position of the MRI image and 3D windows.
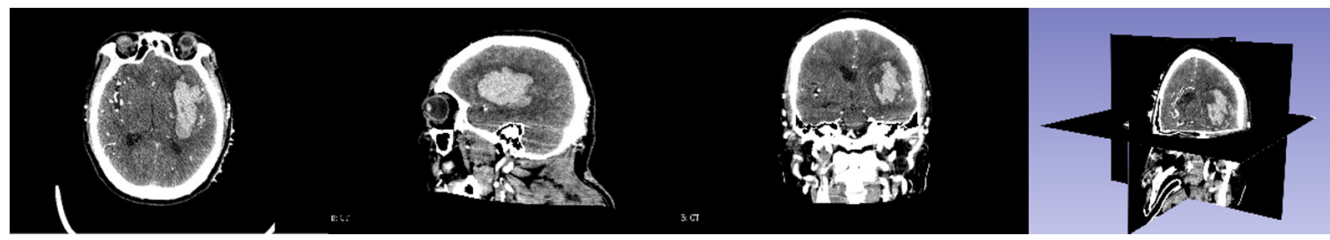

Fig. 1. CT image

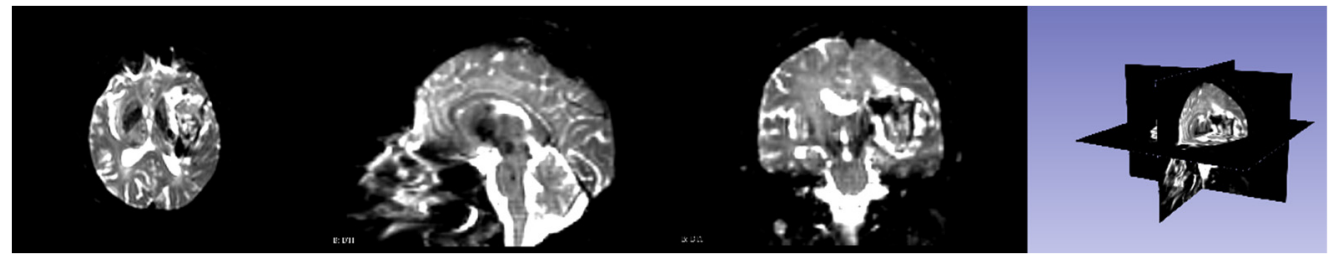

Fig. 2. MRI image

\subsubsection{Image registration}

Although great progress has been made in different medical imaging equipment, and the quality of imaging has also been greatly improved, due to the difference in their respective imaging principles, they cannot be replaced in clinical applications. Medical images with different modalities have their own advantages and characteristics, but the information provided by a single modal medical image is limited. If multiple modal images can be used quickly and efficiently, it can provide doctors with various complementary information of diseased tissues or organs. This kind of complementary information can provide a more comprehensive basis for doctors to diagnose the condition. The best way to solve the above problems is to use multi-modal medical image registration technology. The registration and fusion of medical images of different modalities can provide doctors with a variety of information about diseased organs, which is conducive to the complementarity of information between different modal images, thereby improving the level of medical diagnosis and treatment.

We need to fuse the CT reconstructed hematoma, scalp and MRI reconstructed corticospinal 
tract, so we must register the CT image and MRI image. Elastix is an open source software package that contains a series of algorithms for medical image registration. Most of the code is developed based on ITK. Elastix allows users to quickly test and compare different registration algorithms. The registration effect on CT and MRI images is shown in Fig. 3. The MRI image has $70 \%$ transparency and the CT image has $100 \%$ transparency. It is not difficult to see that the CT and MRI images after registration are basically identical.

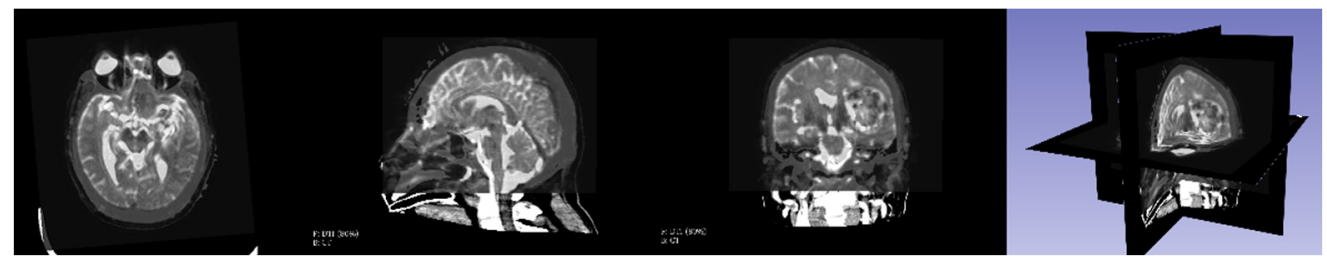

Fig. 3. CT and MRI registration effects

\subsubsection{Three-dimensional reconstruction}

During intraoperative navigation, we need to observe the relative spatial position relationship between the hematoma, corticospinal tract of the patient's brain and the patient's skull, so as to realize the real-time display of the patient's intracranial situation during the operation, so that the operator can clearly observe the surgical instruments spatial positional relationship in the skull. We first modeled the intracranial hematoma of the patient, and processed the CT image through the Watershed function of 3D Slicer. The principle of Watershed is create complete segmentation from seeds, similarly to "Grow from seeds" effect in Slicer core. Advantage is that this effect can enforce smooth surfaces, thereby preventing leaks and reducing the need for additional smoothing after region growing. Disadvantage is that this effect recomputes the complete segmentation after any seed is changed, therefore it is significantly slower. The modeling effect of the hematoma by Watershed is shown in Fig. 4. The yellow part in the figure is the hematoma area from the image threshold, and the red is displayed in the 3D window. A 3D model of the patient's intracranial hematoma came out.

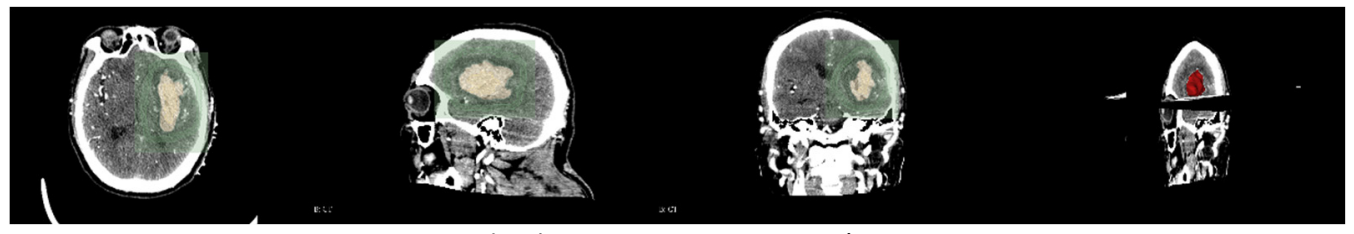

Fig. 4. Hematoma reconstruction

During hematoma or tumor surgery, functional areas and brain proteins should be kept as much as possible to avoid secondary damage caused by surgery. Generally, brain tumors and hematomas are located in the brain parenchyma, and diffusely infiltrate and grow showing diffuse infiltration and growth, and the boundary with normal brain tissue is not clear. Therefore, most traditional surgical resections are performed as far as possible beyond the naked eye, in this way, it is inevitable to damage the functional fiber bundle during the operation, leading to postoperative dysfunction, especially when the lesions are in important functional areas. Therefore, how to not only completely remove the tumor, but also protect the functional area from damage is a key issue. Traditional magnetic resonance imaging (MRI) can clearly display basic information such as the diameter, location, and surrounding edema of intracranial lesions, and can roughly estimate the nature of the tumor, but it can't show the direction of the white matter fiber bundle around the lesion. With the development of computer and magnetic resonance imaging technology, the emergence of diffusion tensor imaging (DTI) technology has enabled the non-invasive study of brain white matter fiber bundles. DTI is an imaging method that uses the changes in magnetic 
resonance signals caused by the diffusion of water molecules in various directions in the brain tissue to detect the microstructure of the brain tissue. Diffusion tensor tractography (DTT) is an imaging technology that is based on magnetic resonance diffusion tensor imaging (DTI). It is the only non-invasive method for displaying white matter fiber bundles in vivo. The advantage of the imaging method is that it can more clearly show the positional relationship between brain white matter fibers and tumors, especially in terms of edema around the lesion and intracranial cross fibers. We first need to perform a mask of the brain on the MRI image data as shown in Fig. 5. It can be seen that the entire brain is masked by the three positions of axis, coronal and sagittal. After the mask, the diffuse fiber bundle imaging technology is used to reconstruct the three-dimensional fiber bundles in the brain as shown in Fig. 6. The relative positional relationship of the hematoma fiber bundles can be clearly observed in the picture, which can effectively avoid the secondary caused by surgery, reduces the risk of surgery and improves the patient's prognosis.

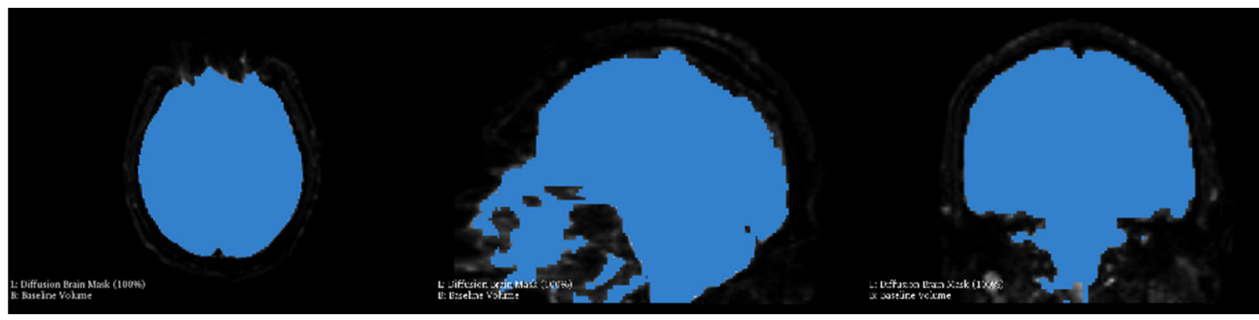

Fig. 5. Brain mask

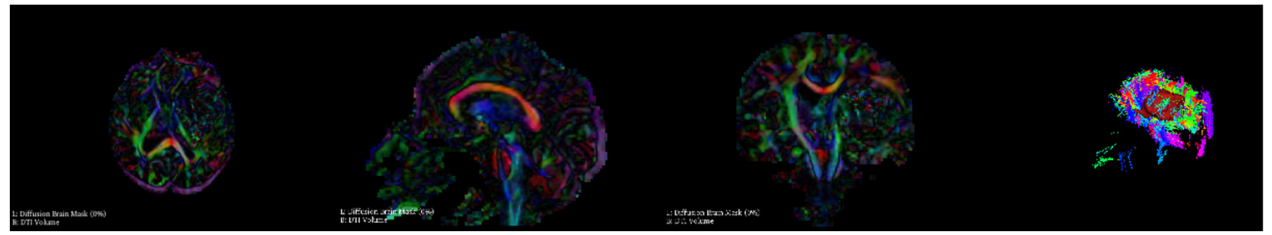

Fig. 6. DTI imaging

CT images are expressed in different gray levels, reflecting the differences in X-ray absorption coefficients of different tissues of the human body, that is, differences in tissue thickness and density. Black shadows indicate low-absorption regions, that is, low-density regions, such as lungs with more gas. White shadows indicate areas of high absorption, that is, areas of high density, such as bones. In addition, the CT image has high density resolution, and the imaging of soft tissues, bones, and joints is relatively clear. It is an ideal inspection method to observe bone, joint, and soft tissue lesions, so we need to use CT images to reconstruct the skull of the patient as shown in the Fig. 7. It is not difficult to see the relative positional relationship of the skull, hematoma, blood vessels and fiber bundles in the figure, which has achieved a good effect.

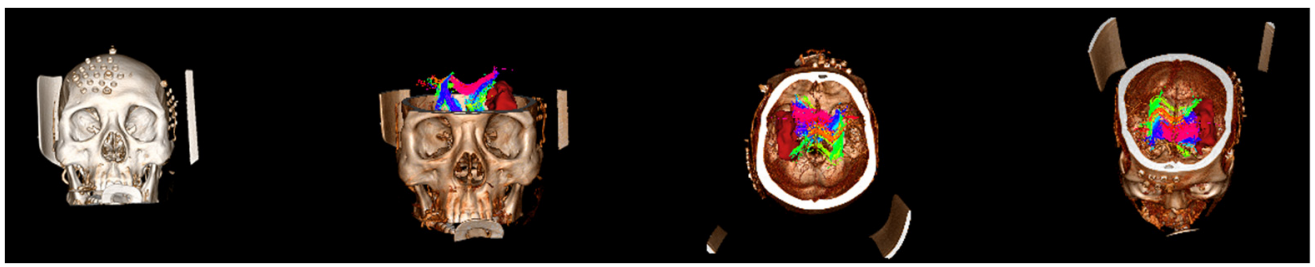

Fig. 7. Skull reconstruction

During the intraoperative navigation stage, the patient's face and surgical instruments need to be registered to prevent errors caused by skin displacement. We apply the 3D Slicer's Threshold method to reconstruct the patient's face. The Threshold method is mainly fill segment in a selected region based on master volume intensity range. The feature size, ROI, and fill method can be 
changed to prevent leaks into other structures. Adjusting the threshold of Threshold to -794.06-1124.99, as shown in Fig. 8, the skull in the CT image can be seen that the brain is completely masked. The masked image is face-modeled as shown in Fig. 9. The first three images are the fat structure of the patient's face, and the last one is the patient's face with $70 \%$ transparency. We can see the location of hematoma, fiber bundle and face, which facilitates the operator's operation during the operation and observation of the location of the surgical instruments. The operation can be completed better, which greatly increases the success rate of the operation.

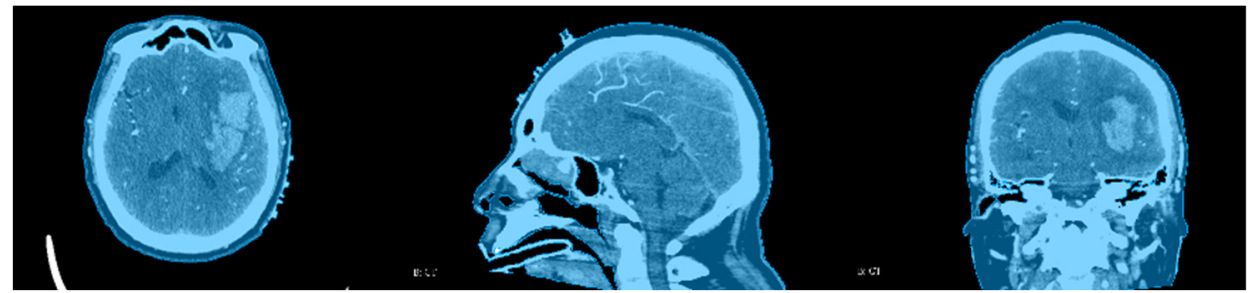

Fig. 8. CT threshold

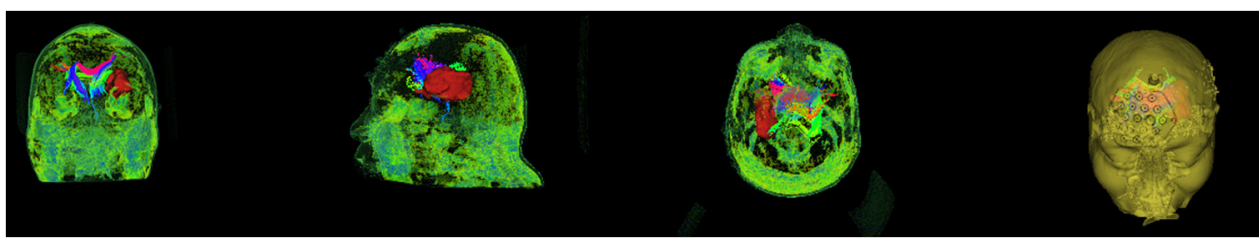

Fig. 9. Face reconstruction

\subsection{Intraoperative navigation}

The intraoperative navigation part is composed of a guiding instrument, a display screen, a real-time positioning tracking navigation system, a surgical needle, etc., and the real-time positioning tracking navigation system is used to display the leision, one dimensioned motion of the surgical instruments when they are in the coronal, sagittal and axial position and relative positional relationship in three-dimensional space in real time. It can transform coordinates of real-time positioning tracking navigation system's own coordinate system and the world coordinate system of the patient entity to complete the registration of surgical instruments, virtual three-dimensional models and patient entity Quasi-sum registration. Then, the surgeon fine-tunes the planned surgical path, and determines to avoid the patient's corticospinal tract and other important fiber bundles and large blood vessels before surgery. During the operation, the virtual model of the focus image and surgical instrument is displayed on the display, and the depth and angle of the catheter or surgical probe entering and the relative spatial position relationship with the calibration target are displayed in real time. The length measurement error is less than $1 \mathrm{~mm}$, and the angle measurement error is less than 3 degrees. It provides real-time surgical navigation in this way.

\subsubsection{NDI passive polaris system}

NDI's Passive Polaris system is the most widely used stereo positioning system in surgical navigation systems. It mainly consists of active receiving optical sensors, a four-point passive infrared reflection reference tool (4-passive reference), and a three-point passive infrared reflection measurement tool (3- passive probe). The structure is shown in Fig. 10. The optical positioning system is an image navigation device produced by the Canadian company NDI. The positioning accuracy is $0.35 \mathrm{~mm}$. It can meet the requirements of the surgical navigation system within the tolerance of $1 \mathrm{~cm}$ in general surgical navigation systems. The optical positioning system 
Polaris uses a binocular camera to track the target, and uses advanced optical measurement technology to track the 3D position and orientation of active or passive markers attached to the surgical tool, providing superior accuracy and reliability. The objective of optical measurement is to calculate the position and orientation of an object or tool within a defined coordinate system. Optical tracking uses position sensors to detect infrared emitting or retroreflective markers attached to a tool or object. The position sensor can calculate the position and orientation of the tool based on the information the position sensor receives from those markers. Active markers are infrared emission markers that are activated by electrical signals, and passive markers are spherical retroreflective markers that reflect infrared light and are emitted by a mirror on a position sensor. The motion tracking system is flexible and easy to operate. As long as it is within the tracking area, it can perform high-precision positioning. It can adapt to movement in different directions and angles. It can also display the measurement results in real time, which is convenient for the operator to observe and give clinical medicine Brings great convenience.

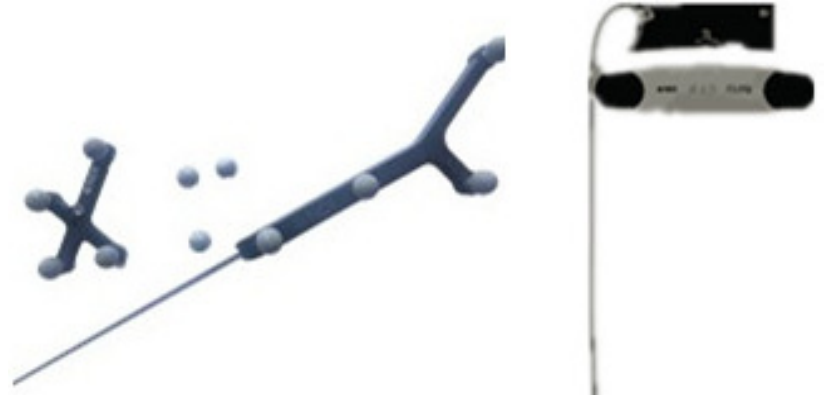

Fig. 10. NDI passive polaris system

\subsubsection{NDI and 3D slicer connection}

The space coordinates of the infrared navigator are converted into the space coordinate data required by the 3D Slicer through a Plus interface as shown in Fig. 11. This interface first needs to create a device set name and description, set the device ID and tool type, and then edit PlusOpenIGTLinkServer Part to define the content to be sent to Slicer. Through the connection of NDI and 3D Slicer, the trajectory of the surgical needle relative to the reference position sensor can be displayed in real time. The reference tool is defined as ReferenceToTracker, and the surgical needle is StylusToTracker. After the connection is successful, the device that can be read within the recognition range of the navigator will be displayed in the 3D Slicer. At the same time, the model of the needle under the StylusToTracker coordinate system will be displayed in the 3D window and the real-time location of the StylusToTracker tool will be shown in the figure. As shown in Fig. 12.

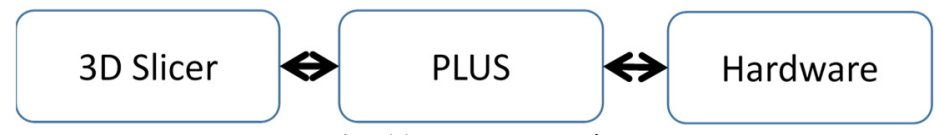

Fig. 11. Data conversion

\subsubsection{Enrollment}

Different devices of the navigation system have their own independent coordinate systems, including model coordinate systems, probe coordinate systems, patient coordinate systems, and camera coordinate systems.

1) Model coordinate system.

The three-dimensional virtual model of the patient reconstructed from multi-modal medical images such as CT / MRI of the patient before surgery. The coordinate system where the model is 
located is the model coordinate system.

2) Camera coordinate system.

In the navigation system, the space positioning device also has its own set of system coordinates, which represent the position changes of the surgical instruments and the patient in the actual operating space in real time, that is, the camera coordinate system.

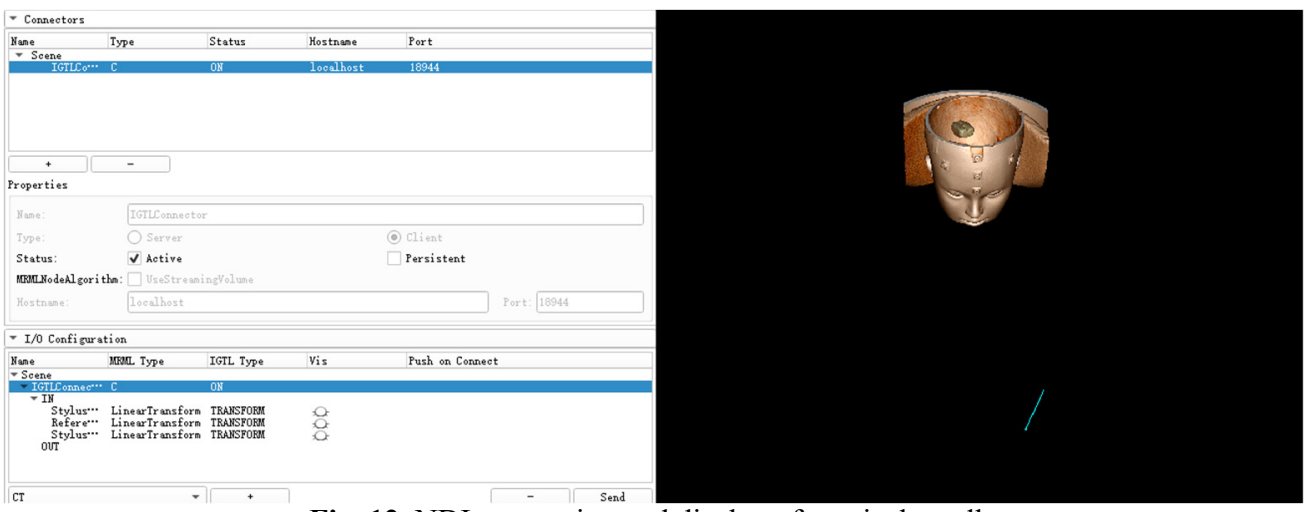

Fig. 12. NDI connection and display of surgical needle

3) Probe coordinate system.

The positioning device of the navigation system obtains the position of the working point at the probe tip by collecting the coordinates of multiple positioning marks of the probe. These positioning marks determine a fixed coordinate system, and the position of the needle point in this coordinate system can be obtained through probe calibration, and then the coordinates of the needle point in the camera coordinate system can be obtained according to the coordinate conversion.

4) Patient coordinate system.

The patient coordinate system is the coordinate system in the patient's reality, which also can be called the world coordinate system. The patient coordinate system generally does not need to know the specific coordinates. The patient coordinate system can be acquired, that is, the coordinates of the patient's body surface marker points acquired by the navigation system in the camera coordinate system. Taking the patient coordinate system as a bridge, the transformation relationship between the camera coordinate system and the model coordinate system can be obtained through spatial registration.

We first register the probe coordinate system, that is, identify the relative spatial position relationship between the needle point and its multiple positioning marks and the change relationship between the positioning marks when the needle body is rotated. The registration errors are $0.299616 \mathrm{~mm}$ and $0.00165287 \mathrm{~mm}$ respectively, as shown in Fig. 13.

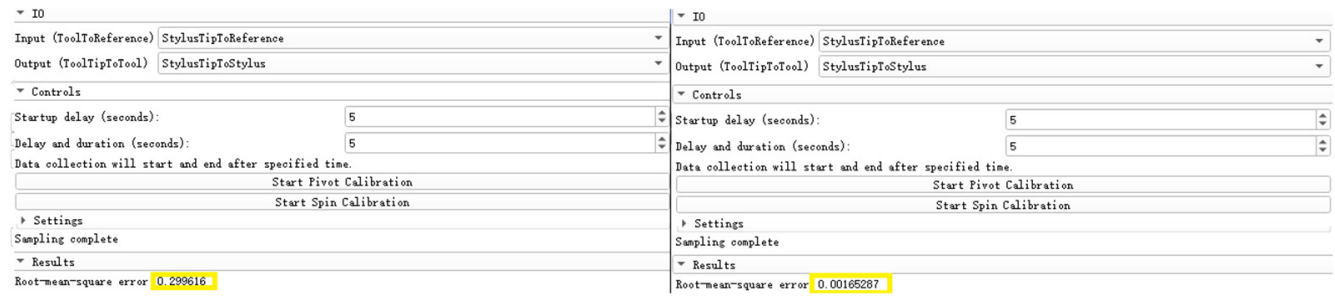

Fig. 13. Probe coordinate system enrollment

The core of the surgical navigation system is to establish a unified coordinate transformation relationship between the image-patient-probe. The transformation relationship between the patient-probe has been completed before. The transformation between the image and the patient 
needs to be realized by intraoperative spatial registration. Spatial registration is a one-to-one correspondence between the actual patient anatomy location and the three-dimensional virtual model in the computer. It is a key step in determining the accuracy of navigation. At present, the common spatial registration methods for surgical navigation systems at home and abroad include: landmark registration, laser registration, mask registration, and anatomical point registration. The marker method has been used since the advent of navigation systems. The main reason is that the marked points can be developed on many images, and the registration process is easy to operate and can be applied to various positions. The laser and mask registration methods cannot be applied to lateral and prone surgery. The anatomical point registration method generally selects points with anatomical marks on the head and face of the external auditory meatus, the root of the nose, and the inside of the clothing, and the registration error is large. Therefore, in this paper, the landmark method is used for spatial registration of surgical navigation. Among the point registration methods, algorithms such as manual registration method, three-point method, unit four-element method, Single Value Decomposition (SVD), and Iterative Closest Point (ICP) are widely used. Apply the three-point method to register the model and the patient, mark the three feature points in sequence on the face model, and then place the surgical needle at the same position on the patient's face in the surgical space in the order of the marked feature points. The position coordinates of the surgical needle tip in model space, so that a point-to-point spatial transformation matrix is established. We then put the probe coordinate system and camera coordinate system under this spatial transformation matrix to complete the registration of unified coordinate transformation between image patient probe. The registration process of the unified coordinate transformation among the users is shown in Fig. 14. The effect and error achieved after the coordinate transformation are shown in Fig. 15. The error 1.74657 can be seen in the figure, and it can be seen that this method also has high accuracy.

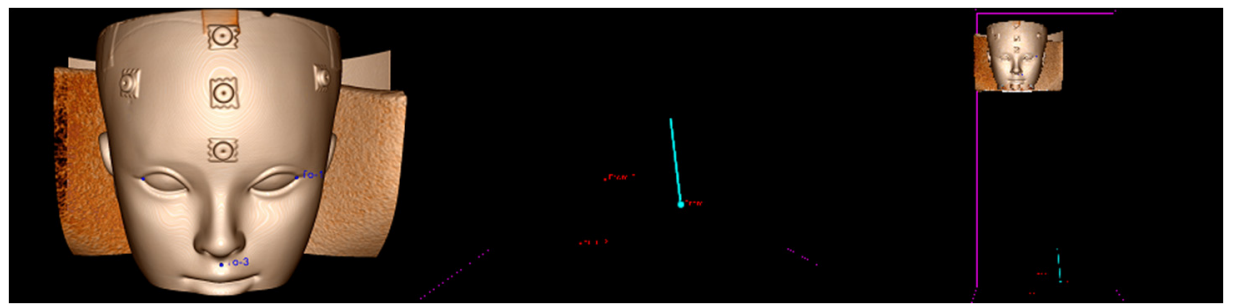

Fig. 14. Model and patient registration
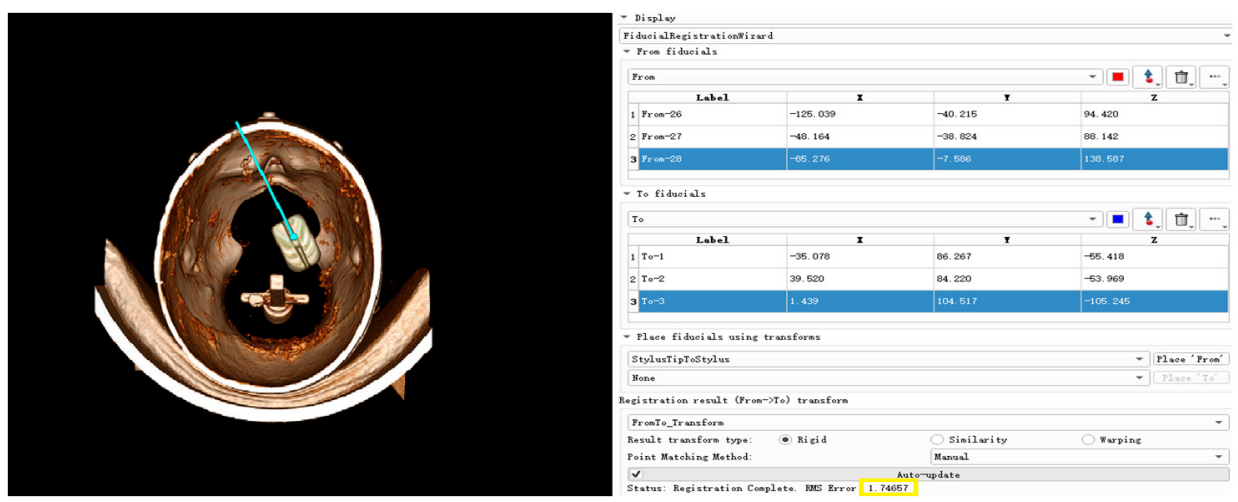

Fig. 15. Registration effect and accuracy

\subsubsection{Surgical path planning}

The brain is the most complicated and important organ in human organs. Therefore, doctors must avoid important tissues and blood vessels in the patient's brain during the operation to ensure 
the success of the operation and the safety of the patient. The preoperative plan requires obtaining image data of the patient before surgery, conducting sufficient research on the lesion area and surrounding tissues, processing the image data according to the requirements of the surgical navigation system, and storing it in a computer for doctors' reference. After completing the 3D reconstruction, the surgical path planning can be performed in the 3D model. The surgical path planning first outlines the lesion area, that is, the target point, in the three-dimensional model, and then determines the puncture point based on anatomy knowledge and doctor's experience and referring to image information. In brain stereotactic surgery, the straight line connecting the puncture point and the target point is the surgical path. At the same time, the system can display the relevant information of the surgical path and the tissue structure within the forward looking distance on the three-dimensional model. As shown, the planned path after the surgical needle approach changes from yellow to red and at the same time marks the distance from the target location, which provides convenience for the operation and avoids risks.

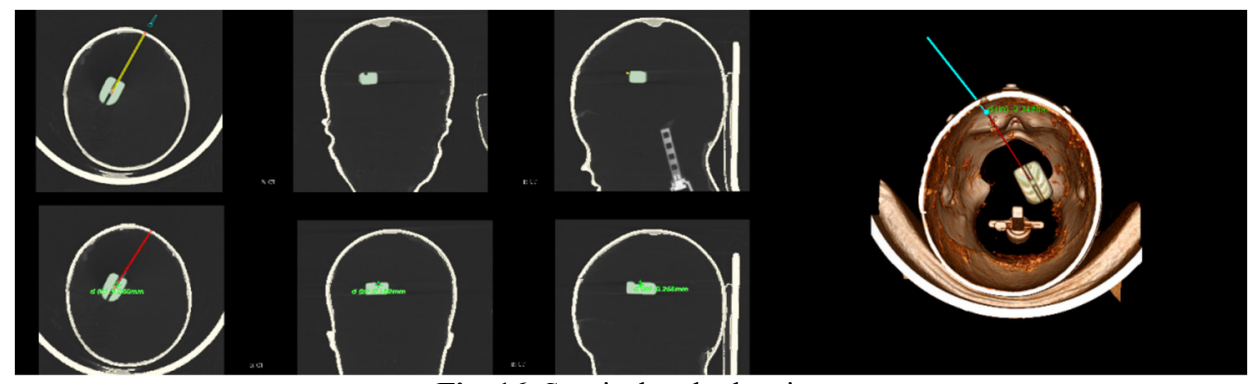

Fig. 16. Surgical path planning

\section{Application of neuronavigation system}

\subsection{Application in the technology of transsphenoidal expansion}

In the surgery of transsphenoidal expansion, neuroendoscopy and microscopy are usually used for assisted surgery. In the operation, the lesion area is too large, and the skull base is significantly damaged. In the surgery of transsphenoidal expansion, it is difficult to identify the tissue structure of the lesion area, resulting in structural damage and residual disease during surgery [25]. With the help of the neuronavigation system, the size of the lesion and the tissue structure near the lesion can be accurately divided. The positional relationship between the lesion and the surrounding tissue can be visually seen and the impact on the surgery can be analyzed. Real time display can feedback important information during the surgery, so that the surgeon can better understand the relationship between important anatomical structure and lesions. The application of neuronavigation can effectively improve the accuracy of surgery and reduce the risk of surgery [26].

\subsection{Application in tumor resection surgery in rock-slope area}

The rock-slope area refers to the area enclosed by the sphenoid bone, the tibia and the occipital bone. It is an important component of the middle cranial fossa and the posterior fossa, usually referred to as the rock slope area [27]. This area is close to many important neurovascular structures, including brain stem, facial nerve, auditory nerve, trigeminal nerve, abducens nerve, vagus nerve, glossopharyngeal nerve and internal carotid artery, posterior communicating artery, posterior cerebral artery and basilar artery [28]. Tumors that occur in the oblique region of the rock tend to be deep and bulky. The anatomical relationship around the tumor is complex, and the blood vessels and cranial nerves are densely distributed. How to avoid the blood vessels and the cranial nerves while removing the tumor completely is especially important [29]. The neuronavigation system can perform tumor boundary division by $\mathrm{CT}$ and MRI images, and 
reconstruct the blood vessels and cranial nerves in three dimensions, visually display the relative positional relationship between the lesions and blood vessels and cranial nerves and can perform optimal surgical path planning before surgery and can display the situation of the surgery in time during the navigation surgery, and effectively improves the safety of tumor resection in the oblique zone and avoids other hazards that may be caused by surgery.

\subsection{Clinical application of glioma in brain functional area}

Glioma is a common neurosurgical malignant tumor disease. It is considered that genetics and environment are the main causes of cancer, which may cause headache, nausea and vomiting, epilepsy, blurred vision and other symptoms, and will affect the corresponding brain function [30]. Glioma is usually treated with surgical resection, which can be difficult for both low-grade gliomas and high-grade gliomas. The principle of surgery emphasizes the maximum retention of patient function. Under the premise, the tumor is removed to the maximum extent, and the degree of tumor resection directly determines the prognosis of the patient. For non-functional tumors, the excision can be performed $1.0-2.0 \mathrm{~cm}$ to the periphery, and for the functional tumor, the resection range should be controlled Outside the ribbon [31]. Usually, in the operation of excising glioma, individual differences and the effects of glioma on the displacement of blood vessels and brain nerve bundles should be considered. The neuronavigation system has the advantages of intraoperative real-time imaging and clear calcification. It can accurately locate the lesions, reduce the damage caused by the surgical incision flap and bone flap design compared with the traditional method, reduce the exposure time to normal brain tissue, and reduce the blind damage to the brain tissue when searching for the lesion [32].

\subsection{Application of descending ventriculo-peritoneal shunt in the treatment of hydrocephalus}

Hydrocephlus is a common neurosurgical disease that enlarges the ventricles and reduces the brain parenchyma due to the imbalance of cerebrospinal fluid secretion and absorption caused by various reasons, or an abnormal accumulation of cerebrospinal fluid in the subarachnoid space or intrauentricular caused by the obstruction of cerebrospinal fluid circulation pathway. Hydrocephalus is often accompanied by elevated intracranial pressure. Its pathogenesis mainly includes cerebrospinal fluid malabsorption, hydrocephalus circulatory disorder and choroid plexus secretion of excessive cerebrospinal fluid. The main treatment for hydrocephalus is ventriculo-peritoneal shunt, originated in 1898 [33]. Surgery inserts the proximal catheter into the lateral ventricle, and the distal catheter is inserted into the abdominal cavity. Generally, it is placed through the frontal horn or occipital angle of the lateral ventricle or the lateral ventricle is placed in the occipital occipital. During the ventriculo-peritoneal shunt ventricle catheterization, neuronavigation can accurately guide the puncture direction and puncture depth, avoiding the damage caused by traditional hand puncture. Using neuronavigation guided puncture ventricle, it can achieve high precision, less trauma and reduce complications. Especially for malformed ventricles and small ventricles, the application of neuronavigation system has a Good effect [34].

\subsection{Application of thalamic hemorrhage into the ventricles}

Thalamic hemorrhage is a common type of hypertensive intracerebral hemorrhage, and its incidence accounts for about $13 \%$ of all hypertensive intracerebral hemorrhage, second only to shell nucleus hemorrhage [35]. The disease is common in patients with hypertension between the ages of 50 and 70, but with the improvement of living conditions, the patients tend to be younger. The thalamic hemorrhage easily breaks into the lateral ventricle, affecting the cerebrospinal fluid circulation pathway, resulting in acute obstructive hydrocephalus, ventricular of the brain swells rapidly, intracranial pressure rises sharply, eventually leading to the formation of cerebral palsy, 
so the thalamic hemorrhage has a higher disability rate and mortality [36]. For deep bleeders, traditional craniotomy can dear hematoma and deboned flap to reduce stress, but the surgical process is very destructive to other brain tissues, and the mortality rate has reached more than $30 \%$ and is still high disability rate [37]. For patients with moderate hemorrhage without cerebral palsy, it can be treated by external drainage of the ventricle. It is safer and better. However, there are also long-term drainage of the drainage tube, which leads to postoperative infection and cannot be straight due to drainage. The shortcomings of secondary hemorrhage caused by hemostasis, so how to completely remove the hematoma and reduce the damage to other tissues during surgery has become an urgent problem to be solved. Neuronavigation technology can plan the surgical path before surgery. Effective way to remove the hematoma, greatly improve the efficiency of surgery, but also reduce the difficulty of surgery, navigation during surgery can clearly show the blood vessels around the hemorrhage, can avoid new bleeding points and other brain tissue during the operation Injury, greatly increased the safety of surgery.

\subsection{Application in epilepsy surgery}

In the field of epilepsy surgery, Kratimenos, etc. [38]. In 1992, the neurosurgical navigation system was first applied to the clinic. At that time, it was called computer-assisted stereotactic selective hippocampal amygdala resection. Focal cortical dysplasia (FCD) is a type of cerebral cortical dysplasia, which is one of the common causes of frontotemporal epilepsy. FCD and language, limb movement, sensory and other functional areas may be adjacent. If functional areas damage, it will be serious the surgical treatment of temporal lobe epilepsy is better, because the hippocampus inside the temporal lobe is the main anatomical structure responsible for memory and cognitive ability. Superior hemisphere temporal lobe resection may cause postoperative speech memory dysfunction [39]. It is more obvious in the dominant temporal lobe, and it is not easy to recover, but the effect on the non-dominant side is relatively small, and it is easy to get compensation. Therefore, it is an important part of surgical treatment of epilepsy to evaluate the language and memory before epilepsy to prevent irreversible damage after surgery. The scope of surgical resection, design of surgical approach to protect important brain functional areas and nerve conduction bundles, and surgical methods are the key content of preoperative evaluation of frontotemporal epilepsy caused by FCD. Neurosurgical navigation system has obvious advantages when it is applied in Ectopic cortical resection, corpus callosum and selective hippocampal amygdala. Under the navigation, the location of the ectopic cortex can be quickly found. The surgeon enters the deep part of the temporal lobe through a well-planned path, and the hippocampus and amygdala are easily identifiable. So, it can avoid damage to the cortex function, safely remove the lesion in the shortest path. It is of great significance to improve the efficacy of epilepsy surgery [40].

\section{Several factors affecting the accuracy of neuronavigation systems}

Although the neuronavigation system can provide a lot of help for traditional surgery, there are often many factors that may affect the accuracy of the neuronavigation system during use.

1) Registration error.

CT and MRI scans are required before the operation. Usually, the CT and MRI scans performed by patients under different conditions often result in non-rigid deformation. Therefore, the obtained CT and MRI data need to be image non-rigid. The effect of non-rigid registration algorithm registration is not as good as that of rigid registration, so it will affect the accuracy of the navigation system.

2) Machine error.

The neuronavigation system is usually a high-precision instrument, which has high requirements on the environment and operation. For example, the optical navigation system may cause inaccurate navigation system due to light problems or density changes of the reflective 
material on the reflective ball. Other types of the navigation system will also affect the accuracy of the navigation system due to the influence of magnetic fields and noise.

3) Enrollment error.

In the intraoperative navigation phase, the surgical instruments, face and $3 \mathrm{D}$ model are first enrolled, usually by point markers or infrared laser 3 enrollment, which may cause enrollment errors due to displacement of scalp shift or unstable infrared signal. The enrollment error will affects the accuracy of the navigation system during surgery.

4) Structural image drift.

During the operation, the craniotomy of the patient's bone flap, the loss of cerebrospinal fluid, the use of anesthesia and dehydrating agent, the traction of surgical instruments, the change of intracranial pressure, the dissolution of hematoma, and the resection of brain tumors may change the internal structure of the patient. The image data that has been scanned will be different. This difference will also affect the accuracy of the navigation system.

\section{Measures to improve the accuracy of the neuronavigation system}

1) Registration.

Registration requires as much as possible to reduce the non-rigid deformation of the patient's CT and MRI data. The two image data required only have a linear relationship of translation, scaling, flipping, rotation, and shear. The best solution is to let the patient to keep the same posture when taking CT and MRI and the time interval between scanning two types of images is very short, and then using the obtained data for rigid registration can achieve good results.

2) Enrollment.

The accuracy of enrollment is related to the location selection of the positioning marker points and the accuracy of the operation. Do not arrange the marker points in a straight line, and do not set the distance of the marker points closer. The best solution is to base it on the three points-the patient's guilt, the outer point and the nose to punctuate in order along the eyebrows and the bridge of nose more than 6 markers. As the marker points increase, the enrollment accuracy is also improved.

3) Structural image drift.

During the operation, try not to use dehydrating agents and excessive ventilation to reduce intracranial pressure. The craniotomy should be as small as possible, and try to avoid loss of cerebrospinal fluid caused by opening the ventricular system and subarachnoid space. We must try to avoid the blood vessels on the surface of the brain, while maintaining the patient's blood pressure balance. After cutting the dura, the intraoperative ultrasound, CT, MRI technology can be used to accurately locate the lesion in real time.

\section{Trends in neuronavigation systems}

Traditional surgery has not been able to meet the needs of a large number of patients to be treated. Instead, it will be a digital, real-time, intelligent neuronavigation system. The birth of neuronavigation is a historic and inter-generational advancement for surgery. Because the emergence of the neuronavigation system reduces the surgical trauma, improves the accuracy of the operation, and improves the reliability and safety of the operation, it does not rely entirely on the surgeon's surgical experience and blind surgery, which improves the success rate of the operation. System equipment is also varied according to needs, and can be combined with other tools as needed, not only in neurosurgery, spine surgery, otolaryngology, but also in orthopedics, radiosurgery, etc., which will determine the neuronavigation system will be the necessary surgical aid in the hospital. Although the development of neuronavigation is very good, it still needs to be improved in some aspects, such as registration accuracy, which will be a breakthrough point in the future research of neuronavigation. 


\section{Acknowledgements}

This work was supported by the National Science Foundation of China $(51674121,61702184)$, Tangshan Innovation Team Project (18130209B,18130210B), the Hebei Key Laboratory of Science and Application, Research and practice project of higher education teaching reform in Hebei Province (2017GJJG111) and Ministry of education production university cooperation education project (201802305012).

\section{References}

[1] American Cancer Society, Surveillance Research, http://www.cancer.org.

[2] Berger M. S., Rostomily R. C. Low grade gliomas: functional mapping resection strategies, extent of resection, and outcome. Journal of Neuro-Oncology, Vol. 34, Issue 1, 1997, p. 85-101.

[3] Berger M. S., Deliganis A. V., Dobbins J., et al. The effect of extent of resection on recurrence in patients with low grade cerebral hemisphere gliomas. Cancer, Vol. 74, Issue 6, 1994, p. 1784-1791.

[4] Keles G. E., Anderson B., Berger M. S. The effect of extent of resection on time to tumor progression and survival in patients with glioblastoma multiforme of the cerebral hemisphere. Surgical Neurology, Vol. 52, Issue 4, 1999, p. 371-379.

[5] Lacroix M., Abi Said D., Fourney D. R., et al. A multivariate analysis of 416 patients with glioblastoma multiforme: prognosis, extent of resection, and survival. Journal of Neurosurgery, Vol. 95, Issue 2, 2001, p. 190-198.

[6] Archip N., Fedorov A., Lloyd B., et al. Integration of patient specific modeling and advanced image processing techniques for image-guided neurosurgery. Medical Imaging 2006: Visualization, Image-Guided Procedures, and Display, 2006.

[7] Kubben PI, Meulen Kj, Schijns O., et al. Intraoperative MRI-guided resection of glioblastoma multiforme: a systematic review. The lancet oncology, Vol. 12, Issue 11, 2011, p. 1062-1070.

[8] Mohyuddin M., Sobani Z. A., Junaid M., et al. Neuronavigation assisted endoscopic sinus surgery. Journal of the Pakistan Medical Association, Vol. 61, Issue 11, 2011, p. 1161.

[9] Ram Z., Cohen Z. R., Harnof S., et al. Magnetic resonance imaging-guided, high-intensity focused ultrasound for brain tumor therapy. Neurosurgery, Vol. 59, Issue 5, 2006, p. 949-956.

[10] Barnett G. H. The role of image-guided technology in the surgical planning and resection of gliomas. Journal of Neuro-Oncology, Vol. 42, Issue 3, 1999, p. 247-258.

[11] Gering D. T., Nabavi A., Kikinis R., et al. An integrated visualization system for surgical planning and guidance using image fusion and an open MR. Journal of Magnetic Resonance Imaging: An Official Journal of the International Society for Magnetic Resonance in Medicine, Vol. 13, Issue 6, 2001, p. 967-975.

[12] Mori S., Frederiksen K., Van Zijl P. C. M., et al. Brain white matter anatomy of tumor patients evaluated with diffusion tensor imaging. Annals of Neurology: Official Journal of the American Neurological Association and the Child Neurology Society, Vol. 51, Issue 3, 2002, p. 377-380.

[13] Nabavi A., Mcl Black P., Gering D. T., et al. Serial intraoperative magnetic resonance imaging of brain shift. Neurosurgery, Vol. 48, Issue 4, 2001, p. 787-798.

[14] Nimsky C., Ganslandt O., Cerny S., et al. Quantification of, visualization of, and compensation for brain shift using intraoperative magnetic resonance imaging. Neurosurgery, Vol. 47, Issue 5, 2000, p. 1070-1080.

[15] Roberts D. W., Hartov A., Kennedy F. E., et al. Intraoperative brain shift and deformation: a quantitative analysis of cortical displacement in 28 cases. Neurosurgery, Vol. 43, Issue 4, 1998, p. 749-758.

[16] Tseng C. S., Chung C. W., Chen H., et al. Development of a robotic navigation system for neurosurgery. Studies in Health Technology and Informatics, Vol. 62, 1999, p. 358-359.

[17] Roberts D. W., Strohbehn J. W., Hatch J. F., et al. A frameless stereotaxic integration of computerized tomographic imaging and the operating microscope. Journal of Neurosurgery, Vol. 65, Issue 4, 1986, p. 545-549.

[18] Watanabe E., Watanabe T., Manaka S., et al. Three-dimensional digitizer (neuronavigator): a new equipment for computed tomography-guided stereotaxic surgery. Surgical Neurology, Vol. 27, 1987, p. 543-547.

[19] Pell M. F. Computer-assisted and frameless stereotaxy in Australia: The operating arm system. Computeras-Assisted Neurosurgery, Springer-Verlag, Tokyo, 1997, p. 11-22. 
[20] Heilbrun M. P., McDonald P., Wiker C., et al. Stereotactic localization and guidance using a machine vision technique. Stereotact Funct Neurosurgery, Vol. 58, 1992, p. 94-98.

[21] Kato A., Yoshimine T., et al. Computer assisted neurosurgery: development of a frameless and armless navigation system (CNS navigator). No Shinei Geka, Vol. 19, Issue 2, 1991, p. 137-142.

[22] Jian W. U. Three-Dimensional High Precise Optical Positioning Technique and its Clinical Application. Tsinghua University, Beijing, 2004, (in Chinese).

[23] Lin Qinyong Accurate Localization and Intervention Methods in Ablation of Liver Cancer Using Optical Surgical Navigation. South China University of Technology, Guangzhou, 2016, (in Chinese).

[24] Xue Yuan Research on Real-Time Computation of Three-Dimensional Digital Image Correlation and Its Biomedical Applications. University of Science and Technology of China, Hefei, 2017, (in Chinese).

[25] Laws E. R., Kanter A. S., Jane J. A., et al. Extended transsphenoidal approach. Journal of Neurosurgery, Vol. 102, Issue 5, 2005, p. 825-828.

[26] Jagannathan J., Prevedello D. M., Ayer V. S., et al. Computer-assisted frameless stereotaxy in transsphenoidal surgery at a single institution: review of 176 cases. Neurosurgical Focus, Vol. 20, Issue 2, 2006, https://doi.org/10.3171/foc.2006.20.2.10.

[27] Safavi Abbasi S., De Oliveira J. G., Deshmukh P., et al. The craniocaudal extension of posterolateral approaches and their combination: a quantitative anatomic and clinical analysis. Operative Neurosurgery, Vol. 66, 2010, p. 54-64.

[28] Liu Feng, Xiao Sanchao, Qian Chao, et al. Comparison of three different surgical methods in the treatment of petroclival meningiomas. Neural Injury and Functional Reconstruction, Vol. 13, Issue 4, 2018, p. 208-210, (in Chinese).

[29] Wang Zhongcheng Wang Zhongcheng Neurosurgery. Hubei Science and Technology Press, Wuhan, 2015, p. 597-599, (in Chinese).

[30] Chang Feng, Wang Shuqin The clinical value of multimodal neural navigation technology combined with electrophysio-logical technique for the surgery of skull base tumors. Neural Injury and Functional Reconstruction, Vol. 12, Issue 3, 2017, p. 218-220, (in Chinese).

[31] Miller J. A., Fabiano A. J. Comparison of operative time with conventional fluoroscopy versus spinal neuronavigation in instrumented spinal tumor surgery. World Neurosurgery, Vol. 105, 2017, p. 412-419.

[32] Zhang Guochen, Zhao Sijun, Liang Zhen, et al. Application of intra-operative continuous neuronavigation in low-grade glioma surgery. Chinese Journal of Practical Nervous Diseases, Vol. 12, 2019, (in Chinese).

[33] Scarff J. E. Treatment of hydrocephalus: an historical and critical review of methods and results. Journal of Neurology, Neurosurgery, and Psychiatry, Vol. 26, Issue 1, 1963, p. 1-26.

[34] Gilard V., Magne N., Gerardin E., et al. Comparison of electromagnetic neuronavigation system and free-hand method for ventricular catheter placement in internal shunt. Clinical Neurology and Neurosurgery, Vol. 158, 2017, p. 93-97.

[35] Zhou Liangfu Modern Neurosurgery. 2nd Edition, Fudan University Press, Shanghai, 2015, (in Chinese).

[36] Zhang Zaiqiang, Li Xingang, Shao Yi, et al. Surgical treatment under neuroendoscopy intraventricular hemorrhage. Chinese Journal of Neurosurgery, Vol. 21, Issue 11, 2005, p. 672-674, (in Chinese).

[37] Shah S. D., Kalita J., Misra U. K., et al. Prognostic predictors of thalamic hemorrhage. Journal of Clinical Neuroscience, Vol. 12, Issue 5, 2005, p. 559-561.

[38] Kratimenos G. P., Pell M. F., Thomas D. G. T., et al. Open stereotactic selective amygdalo-hippocampectomy for drug resistant epilepsy. Acta Neurochirurgica, Vol. 116, Issues 2-4, 1992, p. 150-154.

[39] Verrotti A., D'egidio C., Agostinelli S., et al. Antiepileptic drug withdrawal in childhood epilepsy: What are the risk factors associated with seizure relapse? European Journal of Pediatric Neurology, Vol. 16, Issue 6, 2012, p. 599-604.

[40] Van Roost D., Schaller C., Meyer B., et al. Can neuronavigation contribute to standardization of selective amygdalohippocampectomy? Stereotactic and Functional Neurosurgery, Vol. 69, Issues 1-4, 1997, p. 239-242. 


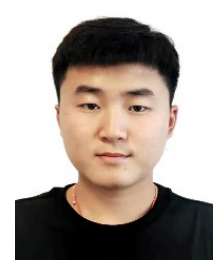

Qi Zheng received his bachelor's degree in information and computation science from North China University of Science and Technology and is now a graduate student in computational mathematics in North China University of Science and Technology. His research fields include theories and methods in B spline, Medical image registration and Medical image reconstruction. In this paper Qi Zheng proposed and implemented a surgical plan for the neuronavigation system, registered the data, reconstructed the three-dimensional model required in the plan. Connected the navigation with the software, registered the patient's face, and was a major contributor in writing the manuscript.

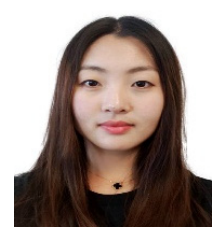

Chaoyue Liu received her bachelor's degree in information and computation science from North China University of Science and Technology and is now a graduate student in computational mathematics in North China University of Science and Technology. Her main research direction is numerical calculation and application. At present, she has some research on track prediction. In this paper Chaoyue Liu summarized and reviewed the literature, and pointed out the prospects and deficiencies.

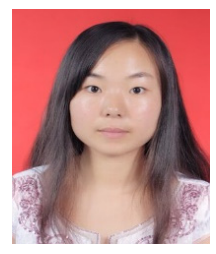

Qiuling Pan received her bachelor's degree in information and computation science from Hebei North University and is now a graduate student in computational mathematics in North China University of Science and Technology. Her research fields include theories and methods in numerical approximation and B spline. In this paper Qiuling Pan summarized and reviewed the literature, and pointed out the prospects and deficiencies.

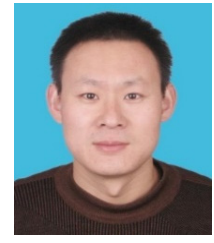

Jincai Chang received his B.Sc. degree in 1996 from Ocean University of China, received his M.Sc. degree in 2005 from Yanshan University, received his Ph.D. degree in 2008 from Dalian University of technology, now he is Professor in North China University of Science and technology. His main research interests include theories and methods in mathematical modelling and scientific computation, numerical approximation and computational geometry, etc. In this paper Jincai Chang put forward the question of the research and approved the final manuscript.

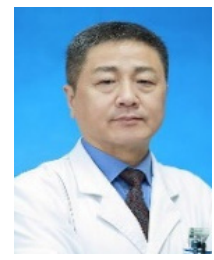

Jianzhong Cui received his Ph.D. degree in 1997 from Tianjin Medical University, now he is Vice President and chief doctor of neurosurgery department of Tangshan Gongren Hospital, and Professor in Hebei Medical University. In this paper Jianzhong Cui put forward the question of the research and approved the final manuscript. 